\title{
Cancer Treatment Induced Bone Loss (CTIBL)
}

\author{
Laura Boehnke Michaud, Susan Goodin
}

\section{Source}

Laura Boehnke Michaud, Susan Goodin. (2006). Cancer-treatment-induced bone loss, part 1. doi:10.2146/ajhp050045.p1.

CTIBL is a long-term complication associated with cancer therapies that can directly or indirectly affect bone metabolism. Although CTIBL can occur in any patient receiving a cancer therapy known to cause bone loss, CTIBL is most common in patients with breast or prostate cancer who receive chemotherapy, hormone therapy, or surgical castration. CTIBL causes bone fragility and an increased susceptibility to fractures; 